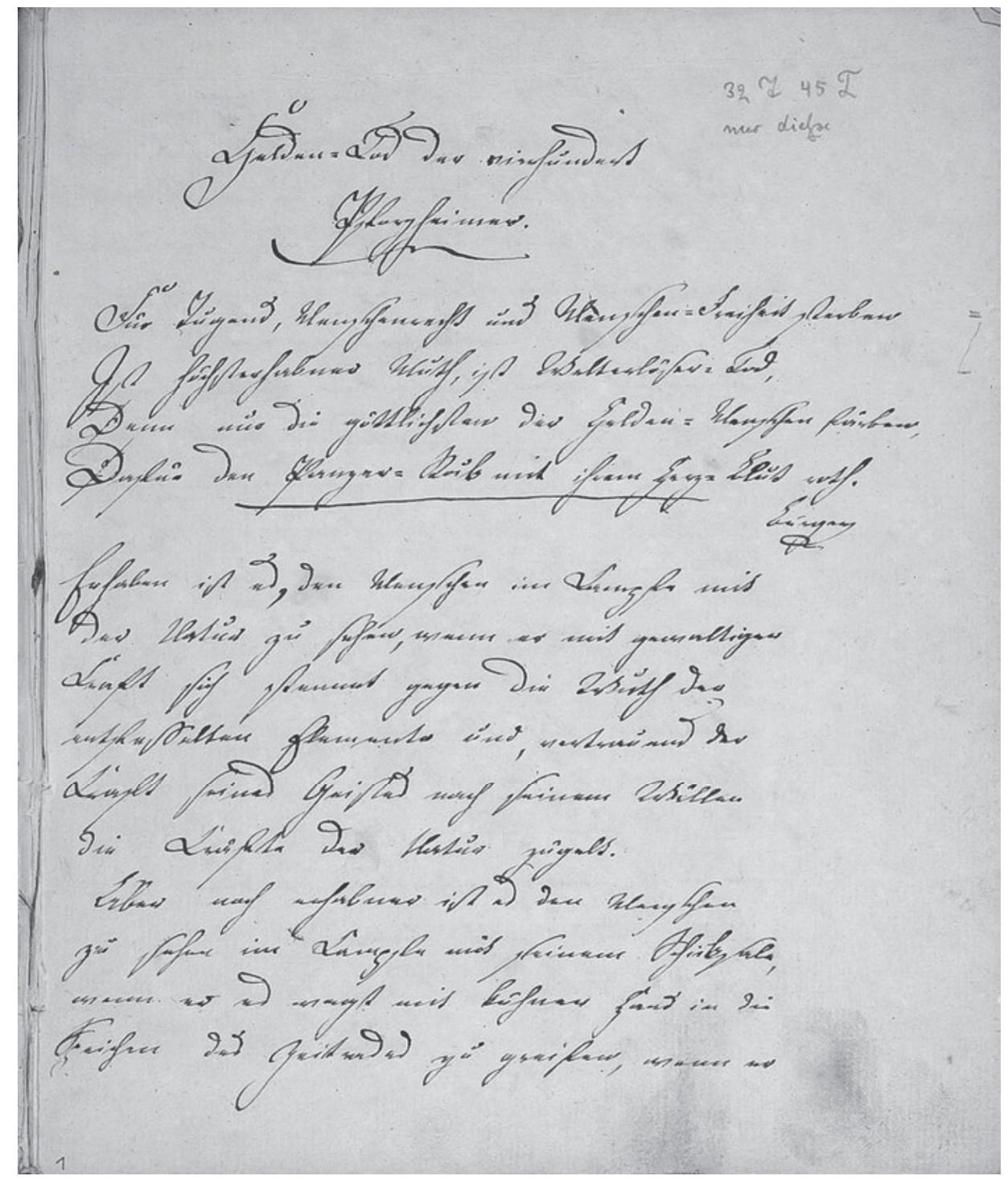

Abb. 1: Georg Büchners Rede „Helden-Tod der vierhundert Pforzheimer“: eigenhändige Reinschrift in einem mit angeklebtem Pappeinband versehenen Heft zu 23 Blatt in zwei Lagen im Quartformat, $170 \times 200$ mm, 1829/30, Weimar, Goethe- und Schiller-Archiv, Inv. Nr. 10/14. 


\title{
Georg Büchner: „Helden-Tod der vierhundert Pforzheimer“ (1829)
}

\author{
Achim Aurnbammer
}

Georg Büchners Schulrede über den „Helden-Tod der vierhundert Pforzheimer" aus dem Jahre 1829 (Abb. 1) ist ein wenig beachtetes, gleichwohl exemplarisches ,Objekt des Heroischen'. Diese Rede verfasste der sechzehnjährige Büchner im Deutschunterricht der Selecta, der letzten Schulklasse am Pädagog, dem großherzoglichen humanistischen Gymnasium in Darmstadt, das er von 1825 bis zum erfolgreichen Abschluss im März 1831 besuchte. ${ }^{1}$ Georg Büchner wurde zwei Mal die Ehre zuteil, bei den Feierlichkeiten zum Ende eines Schuljahres solche schriftlich ausgearbeiteten Reden auch öffentlich vorzutragen: Erhalten ist seine cäsarkritische Rechtfertigungsrede für Cato Uticensis aus dem Sommersemester 1830, während seine politische „Rede des Menenius Agrippa an das römische Volk auf dem heiligen Berge" aus dem Wintersemester 1829/30 nicht auf uns gekommen ist. Ob Büchner die Rede vom „Helden-Tod der vierhundert Pforzheimer" öffentlich vorgetragen hat, ist nicht bekannt.

Die Kenntnis des Themas, einer Episode aus dem Dreißigjährigen Krieg, verdankt Büchner dem Schulunterricht am Darmstädter Gymnasium. So findet sich Büchners Hauptquelle, eine Gedenkrede des Karlsruher Historikers Ernst Ludwig Posselt von 1788, als Teildruck im „Lehrbuch der teutschen prosaischen und rednerischen Schreibart" von Karl Heinrich Ludwig Pölitz (1827), das die Grundlage für die „Theorie der Stylgattungen“ im Darmstädter Deutschunterricht bildete. ${ }^{2}$

1 Vgl. Roland Borgards / Harald Neumeyer: Büchner-Handbuch. Leben - Werk - Wirkung, Stuttgart/Weimar 2009, s. v. „Schriften aus der Schulzeit“, hier 1.4 („Helden-Tod der vierhundert Pforzheimer"), S. 2-4. Siehe Osterprogramm 1830. Büchner hatte zu seinem Deutschlehrer Karl Baur (1788-1877) allerdings ein schlechtes Verhältnis.

2 Ernst Ludwig Posselt: Dem Vaterlandstode der vierhundert Bürger von Pforzheim [Bruchstück], in: Karl Heinrich Ludwig Pölitz: Lehrbuch der teutschen prosaischen und rednerischen Schreibart für höhere Bildungsanstalten und häuslichen Unterricht, Halle 1827, S. 297-303. Hingewiesen auf diesen Teilabdruck hat zuerst Gerhard Schaub: Die schriftstellerischen Anfänge Georg Büchners unter dem Einfluß der Schulrhetorik, Bd. 1, im Fachbereich II (Sprach- und Literaturwissenschaften) der Universität Trier als Habilitationsschrift vorgelegt, Trier 1980, S. 120 (unveröffentlichtes Typoskript). Vgl. auch den Kommentar im Quellenteil der Marburger Ausgabe: Georg Büchner: Sämtliche Werke und Schriften: historisch-kritische Ausgabe mit Quellendokumentation und Kommentar (Marburger Ausgabe), Bd. 1, 2; Bd. 2, 2, Schülerschriften und Schulhefte [künftig: MA], hrsg. von Susanne Lehmann, Darmstadt 2013, S. 233, demzufolge aber ein vollständiger Druck und nicht dieser Teilabdruck Büchners Quelle gewesen sei. Pölitz’ „Lehrbuch“ bildete wohl die Grundlage für die im Deutschunterricht „fortgesetzte Erklärung der Theorie der Stylgattungen“ (vgl. Osterprogramm 1830). Vgl. Borgards /Neumeyer: Büchner-Handbuch (Anm. 1), S. 2. 
Überliefert ist der Schulaufsatz als 28-seitiges, eigenhändig betiteltes Manuskript im üblichen Quartformat. Gebunden wurde es nachträglich als unpaginiertes Heft mit Pappeinband. ${ }^{3}$ Den Aufsatz - sichtlich eine Reinschrift mit typischen Abschreibfehlern - verwahrt das Goethe-Schiller-Archiv Weimar. Erstmals ediert wurde Büchners Rede vom „Helden-Tod der vierhundert Pforzheimer“ 1922, seit 2011 liegt sie in diplomatischer Umschrift und einer umfangreichen Quellendokumentation in der sogenannten Marburger Ausgabe vor. ${ }^{4}$

Mittlerweile hat die Forschung die Quellen zur „Helden-Tod“-Rede präzisiert, doch fehlt bis heute eine Untersuchung, welche die sprachlich-stilistische Faktur von Büchners Rede würdigt und deren ,Dialogizität bewertet, das heißt die produktive Bearbeitung der Prätexte, um Büchners Entwurf eines Heldenkollektivs genauer zu konturieren. ${ }^{5}$ Büchners Rede ist nach klassischem Muster viergegliedert: in exordium, narratio, argumentatio und peroratio, - Einleitung, Erzählung, Beweisführung und Schluss. Einleitend erklärt Büchner, dass nicht die „bloße That" und ihre "Wirkung“ die Bewertungskriterien heroischen Handelns seien, „sondern [...] Motive und [...] Umstände [...], welche eine solche That bewirkten, begleiteten und bestimmten" ${ }^{6}{ }^{6}$ Zudem sei Heroik kein Privileg der Antike, sondern komme auch in der Gegenwart vor. Dies zeige die heroische Selbstversenkung des „Vainqueur“, eines zur Kapitulation aufgeforderten Schiffes der französischen Revolutionsflotte im Seekrieg gegen die Engländer. Büchners Beispiel verrät den revolutionären Gehalt seiner Rede, denn die Selbstversenkung des französischen Schlachtschiffs Vengeur im Jahre 1794 wurde bald zu einem Mythos der Revolutionsmarine und in einen kollektiven Heldentod umgedeutet: Nicht nur ein kleiner Teil der Besatzung, sondern die gesamte Mannschaft wäre mit dem Ruf „Vive la Patrie, vive la République!“ („Es lebe das Vaterland, es lebe die Republik!") untergegangen, und ein Schiffsmodell im Pantheon zeugt noch heute von der Persistenz der Heldenlegende. ${ }^{7}$ Dass Büchner in seiner Rede den eigentlichen Schiffsnamen ,Vengeur' (,Rächer [des Volkes] ${ }^{6}$ ) zu ,Vainqueur ${ }^{6}$ (,Sieger') verballhornt, bekundet seine Parteinahme für diesen revolutionären Freitod, denn schon

3 Vgl. Goethe- und Schiller-Archiv Weimar, GSA 10/14 $(200 \times 170 \mathrm{~mm})$.

4 Vgl. MA, Bd. 1, 2, S. 6-75, S. 215-259.

5 Vgl. Sandra Holzheimer: „Riesenbild“ - Figuration und Defiguration des Heroischen bei Büchner, in: Nikolaus Immer / Mareen van Marwyck: Ästhetischer Heroismus. Konzeptionelle und figurative Paradigmen des Helden (Edition Kulturwissenschaft; 22), Bielefeld 2013, S. 149-173.

6 MA, Bd. 1, 2, S. 8.

7 Vgl. dazu Thierry Roquincourt: Le mythe du Vengeur, in: Michel Vovelle (Hrsg.): Révolution et République - L'exception française, Paris 1994, S. 479-495; Herbert Schneider: Le mythe du vaisseau ,Le Vengeur' de 1794 à 1951, in: Acta Musicologica 77, 2005, S. 71-121. Die Interpretatio nominis von ,Vengeur' als ,Vainqueur' findet sich etwa in der hymnischen Poetisierung der Episode im „Chant des victoires“ von Marie-Joséphe Chenier: „Lève-toi, sors des mers profondes, / Cadavre fumant du Vengeur! / Toi qui vis les Français vainqueur / Des Anglais, des feux et des ondes!“, vgl. Marie-Joseph Chénier: Euvres, Bd. 3, Paris 1824, S. 372-375, hier S. 373. 
die Revolutionspropaganda hatte dieses sprachspielerische Umdeutung vorgeprägt. ${ }^{8}$

Die narratio berichtet von einer vaterländischen Heldentat der Neuzeit, dem kollektiven Opfertod von vierhundert Pforzheimer Bürgern. Sie hätten bei der Schlacht von Wimpfen im Mai 1622 den Rückzug ihres protestantischen Heerführers, des Markgrafen Georg Friedrich von Baden, gedeckt, als dieser fliehen musste, nachdem seine Pulverwagen getroffen worden waren. Indem die vierhundert Pforzheimer, angeführt von ihrem Bürgermeister Deimling, sich im hoffnungslosen Kampf gegen den übermächtigen Feind aufopferten, hätten sie verhindert, dass Tilly mit seinem Heer dem flüchtigen Markgrafen nachsetzen konnte.

In der Beweisführung würdigt Büchner den kollektiven „Helden-Tod“ der Pforzheimer Bürger, indem er ihn über vergleichbare antike Gruppenhelden stellt: die vierhundert Römer, die sich im Punischen Krieg der karthagischen Armee opferten und so dem römischen Heer den Rückzug ermöglichten, wie auch die dreihundert Spartaner, die bei den Thermopylen den Vormarsch der Perser durch ihren Opfertod verhinderten. Diesen antiken Heldenkollektiven seien die modernen Gruppenhelden, die Pforzheimer Bürger, insofern überlegen, als sie zum einen „freie Wahl“ hatten - „und sie wählten den Tod“ - und zum andern für die Nachwelt, „für die ungebornen Enkel“, einen „Welterlöser-Tod“ starben. ${ }^{9}$ Diesen Terminus verdankt Büchner Gottfried August Bürgers Gedicht „Die Tode“ (1793), dessen erste Strophe er als Motto seiner Rede vorgeschaltet hat ${ }^{10}$ - die Leitbegriffe der Französischen Revolution sind nicht zu überhören:

„Für Tugend, Menschenrecht und Menschenfreiheit sterben

Ist höchst erhabner Mut, ist Welt-Erlösertod:

Denn nur die göttlichsten der Heldenmenschen färben

Dafür den Panzerrock mit ihrem Herzblut rot.

Am höchsten ragt an ihm die große Todesweihe

Für sein verwandtes Volk, sein Vaterland hinan.

Dreihundert Sparter ziehn in dieser Heldenreihe

Durchs Tor der Ewigkeit den übrigen voran. "11

8 Vgl. Ilona Broch: Drei Marginalien zu Georg Büchners Schülerschriften, in: Burghard Dedner / Thomas Michael Mayer (Hrsg.): Georg Büchner Jahrbuch 5, Frankfurt am Main 1985, S. 286-291.

9 MA, Bd. 1, 2, S. 9, Z. 19-20, Z. 42 und S. 10, Z. 2-3.

10 Die erste Strophe dieses Gedichts findet sich auch in der im Darmstädter Unterricht verwendeten Anthologie von Karl Wagner (Hrsg.): Teutsche Geschichten aus dem Munde teutscher Dichter, Darmstadt 1831, als Motto der Ballade „Gustav Adolf“ des „Ehrhardt“. Vgl. Stellenkommentar der MA, Bd. 1, 2, S. 299.

11 Gottfried August Bürger: Die Tode, in: Musenalmanach für 1793. Wieder in: Gottfried August Bürger: Sämtliche Werke, hrsg. von Günter und Hiltrud Häntzschel, München/ Wien 1987, S. 385. Zu dem Gedicht vgl. Walter Grab: Gottfried August Bürger als literarischer Wegbereiter und politischer Weggefährte des deutschen Jakobinismus, in: Wolfgang Beutin / Thomas Bütow (Hrsg.): Gottfried August Bürger (1747-1794), Frankfurt am Main [u.a.] 1994, S. 9-24. 
Bürgers zweite Strophe überhöht mit dem Exempel der dreihundert Spartaner den Opfertod für das Vaterland zu einem Sterben in der Nachfolge Christi. Als ,Heldentod' verbucht Bürger, obzwar den Idealen der Französischen Revolution verpflichtet, auch den „Tod für einen guten Fürsten“, während er den „Soldatentod“, für „bloße Majestät“ zu verbluten, als schmählich abtut. ${ }^{12}$

In der abschließenden peroratio hebt Büchner die Größe der Tat kontrastiv hervor, indem er das kollektive Vergessen der Heldentat beklagt. Dieses Missverhältnis wird empathetisch gesteigert durch eine imaginäre Rede der gefallenen Pforzheimer. Sie richten an die modernen Zeitgenossen die rhetorische Frage, ob sie es beim „Andenken eurer Vorfahren“ zuließen, dass „die Nachkommen freier Männer zu Sclaven werden“ und fordern auf, sich zu entscheiden: „Dort liegt Gold neben Fesseln, hier der seltne Ruhm zugleich die stärkste und beste Nation zu seyn. Wählet."13

Büchner heroisiert den Opfertod der Pforzheimer Bürger auch sprachlich-stilistisch. Jede Heroisierung bedarf der Tat, doch was wäre die Tat ohne die adäquate sprachliche Wiedergabe? Zu Büchners rhetorischer Heldentat gehören zahlreiche Wiederholungsfiguren, Wort- und Satzantithesen, welche die Rede stark pathetisieren. So intensiviert bereits eine im Komparativ variierte Anapher zum Zwecke einer distinctio den Anfang: „erhaben ist es, den Menschen im Kampfe mit der Natur zu sehen [...]. Aber noch erhabner ist es den Menschen zu sehen im Kampfe mit seinem Schicksale [...]."14

Zum andern verleiht Büchner seiner heroischen Rede von den Helden einen Grad größerer Direktheit, indem er an einigen Stellen die Erzähldistanz verringert, den Wahrnehmungs- und Bewusstseinshorizont der Figuren teilt, aus dem distanzierenden Imperfekt ins Präsens wechselt und als Erzähler am Geschehen partizipiert. So etwa, wenn er in einer dreifach gesteigerten dubitatio, einer rhetorischen Frage in erster Person, unverkennbar am erzählten Stoff teilnimmt:

„Doch warum greife ich denn nach außen um solche Männer zu suchen, warum beachte ich denn nur das Entfernte, warum nicht das, was mir am nächsten liegt? Sollte denn mein Vaterland, sollte denn Teutschland allein nicht Helden zeugen können?“15

Durch rhetorische Nachbildung der Heldentat will Büchner sein Publikum zur Anteilnahme am Heroischen bewegen. Daher schärft er die Schlachtbeschreibung ganz auf die kritische Situation zu und legt das Gewicht auf die Heldentat, die sinnfällig vergegenwärtigt wird. Durch gezielte Mimesis-Technik versetzt der Redner sich und sein Publikum in die Lage des Augenzeugen: Präsens als Beschrei-

12 Zur Verflechtung von Opfertod fürs Vaterland und metaphysischer Sinngebung in Gottfried August Bürgers Gedicht „Die Tode“ vgl. Anuschka Albertz: Exemplarisches Heldentum. Die Rezeptionsgeschichte der Schlacht an den Thermopylen von der Antike bis zur Gegenwart (Ordnungssysteme; 17), München 2006, S. 172-174.

13

14 Ebd.

15 MA, Bd. 1, 2, S. 6, Z. 36-39. 
bungstempus, affektische Figuren, intensivierende Detaillierungen, emphatische Wiederholungen, Deiktika („hier“ vs. „dort“) und eine asyndetische Aneinanderreihung von Aussagesätzen dramatisieren und veranschaulichen die Situation auf dem Schlachtfeld.

Bevor aber das Heldenkollektiv wie ein deus ex machina auf den Kriegsschauplatz tritt und sein Leben für den Fürsten opfert, hebt Büchner den Markgrafen Georg Friedrich als „das Muster eines Fürsten“16 namentlich hervor. Seine Schilderung leitet die Heldentat der Pforzheimer Bürger ein, die - eine traditionelle rhetorische Technik - zunächst unerwähnt bleiben, um die Spannung zu erhöhen. ${ }^{17}$ Das Kriegsgeschehen bei Wimpfen wird zum Zwecke der evidentia zunächst im Fürsten personalisiert. Das anfängliche Kriegsglück („Schon lächelt der Sieg [...], schon wähnt sich Friedrich die Helden-Schläfe mit dem blutigen [...] Lorbeer schmücken zu können" ${ }^{18}$ ) schlägt mit der dramatischen Explosion der Pulverwagen ins Gegenteil um: So illustriert eine dreifache gradatio die Not des Fürsten. Sie wird durch das anaphorische Adverb „Vergebens“ verdeutlicht und gipfelt in einer dubitatio, welche die Aporie des Fürsten empathetisch vermittelt:

„Vergebens sucht Friedrich die Seinigen wieder zu sammeln, vergebens erfüllt er zu gleicher Zeit die Pflichten des Feldherren und des Soldaten, vergebens stürzt er sich selbst dem andringenden Feinde entgegen. Von der Übermacht gedrängt muß er endlich weichen und das blutige Schlacht-Feld seinem glücklichen Gegner überlassen. Doch wohin soll er sich wenden?"19

Die anaphorischen Fokuspartikeln ,schon' betonen die akute Gefahr. Umso stärker hervorgehoben wird dadurch der Umschwung im korrespondierenden Temporalsatz („da werfen sich [...]“), dessen betonte Konjunktion ,da' den unerwarteten Einsatz der Pforzheimer Bürger einleitet:

„Schon ist er von allen Seiten umringt, schon überwältigt der Feind den letzten schwachen Widerstand [...]. Da werfen sich vierhundert Pforzheimer, an der Spitze ihren Bürgermeister Deimling dem Feinde entgegen, mit ihren Leibern decken sie, ein unerschütterliches Bollwerk, ihren Fürsten und ihre Landsleute. “20

Die entmenschlichende Metapher „Bollwerk“ wird in der nachfolgenden Beschreibung von Heldentat und -tod wieder aufgenommen sowie in den architektonischen Metaphern „Mauern“ und „Thurm“ variiert. Die Singularform der Metapher ,Bollwerk‘ unterstreicht die unauflösliche Bindung des Heldenkollektivs:

„Tausende stürmt der erbitterte Feind gegen das heldenkühne Häuflein, doch tausende brechen sich an der ehernen Mauer. Unerschütterlich stehen die Pforzheimer, kein[e] Wuth, keine Verzweiflung nur hohe Begeistrung und Todesverachtung mahlt sich in ih-

16 MA, Bd. 1, 2, S. 7, Z. 22.

17 Vgl. Leonid Arbusow: Colores Rhetorici. Eine Auswahl rhetorischer Figuren und Gemeinplätze als Hilfsmittel für akademische Übungen an mittelalterlichen Texten, Göttingen 21963, S. 121.

18

19 MA, Bd. 1, 2, S. 8, Z. 8-13 (Kursivierungen A. Aurnhammer).

20 MA, Bd. 1, 2, S. 8, Z. 13-19. 
ren Zügen. Unablässig stürmt der Feind seine Schlachthaufen heran; doch das Vaterland steht auf dem Spiele, Freiheit oder Knechtschaft ist die große Wahl, keiner weicht, keiner wankt, wie Löwen streiten sie von ihren Leichenhügeln herab, Mauern sind ihre Reihen, ein Thurm jeder Mann, ein Bollwerk von Leichen umgibt sie. Endlich von allen Seiten angegriffen, erdrückt von der Uebermacht, sinken sie Mann an Mann unter Hügeln erschlagner Feinde nieder und winden sich sterbend die unvergängliche Lorbeer-Krone des Siegers und die unsterbliche Palme des Martyre[r]s um die Heldenschläfe. ${ }^{21}$

Das gegnerische Heer, zuvor schon als „Söldner“, „wahnsinnige Fanatiker“ und „mordgewohnte Banden“ pejorisiert, bleibt anonym und wird mit den Kollektiva „Feind“ oder „Gegner" bezeichnet. Dessen hyperbolisch veranschaulichte numerische „Übermacht“ („Tausende“, „Schlachthaufen“) kontrastiert mit der im Diminutiv akzentuierten zahlenmäßigen Kleinheit der heroischen Pforzheimer („das heldenkühne Häuflein“). Doppelte und alliterierende Negationen („keine Wuth, keine Verzweiflung“ und „keiner weicht, keiner wankt“) verstärken als Oppositionsfiguren die nachgestellten positiven Aussagen („nur hohe Begeisterung und Todesverachtung“ und „wie Löwen streiten sie von ihren Leichenhügeln her$a b$ "). Die exzeptionelle heldenhafte Tat eines Kollektivs gewinnt auch im Sterben rhetorische Gestalt im Polyptoton „Mann an Mann“. Den Schluss bildet ein weltlich-metaphysisches Paradoxon, indem die Erschlagenen - dem „WelterlöserTod" entsprechend - einerseits die „Lorbeer-Krone des Siegers“ erhalten, andererseits zu Märtyrern verklärt werden.

Die hochrhetorisierte Ausarbeitung des heroischen Themas verdankt der junge Büchner nicht nur seiner eigenen Sprachbegabung, sondern ganz wesentlich, wie die Forschung inzwischen ermittelt hat, fremden Texten. So weist das letzte Drittel der Rede, die argumentatio und die persuasive peroratio, große Anleihen an Johann Gottlieb Fichtes „Reden an die deutsche Nation“ von 1808 auf.22 Fichte geschuldet sind die nationalen Thesen vom Verdienst der Deutschen für die Entwicklung der Menschheit und vom Protestantismus als Ursprung der Aufklärung. Dagegen folgt Büchners „Detailschilderung des legendären Opfertodes der Pforzheimer Bürger und das oratorische Pathos“ ${ }^{23}$ wesentlich der Gedenkrede „Dem Vaterlandstod der Vierhundert Bürger von Pforzheim“ (Abb. 2), die der badische Historiker Ernst Ludwig Posselt im Karlsruher Schauspielhaus am 29. Januar 1788 im Auftrag und Beisein des Badischen Markgrafen gehalten hat. ${ }^{24}$

21 MA, Bd. 1, 2, S. 8, Z. 21-33.

22 Johann Gottlieb Fichte: Reden an die deutsche Nation, Berlin 1808 (2. Aufl. 1824). Auf Büchners Fichte-Lektüren hingewiesen hat erstmals Werner R. Lehmann: Prolegomena zu einer historisch-kritischen Büchner-Ausgabe, in: Maria Honeit / Matthias Wegner (Hrsg.): Gratulatio. Festschrift für Christian Wegner zum 70. Geburtstag am 9. September 1963, Hamburg 1963, S. 190-225, hier S. 198-206. Im „Helden-Tod“ übernimmt Büchner aus der 8. Rede unter anderem einen zusammenhängenden Passus von mehr als zwanzig Zeilen (im Druck der MA), der 14. Rede verdankt er fast den kompletten Schluss. Vgl. Quellenbericht der MA, Bd. 1, 2, S. 215-259, hier S. 215.

24 Ernst Ludwig Posselt: Dem Vaterlandstod der Vierhundert Bürger von Pforzheim. Eine Rede den 29. Januar 1788. In Gegenwart des Hochfürstlichen Hauses gehalten, Karlsruhe 1788. Wiederabdrucke in: Ernst Ludwig Posselt (Hrsg.): Wissenschaftliches Magazin für Aufklä- 


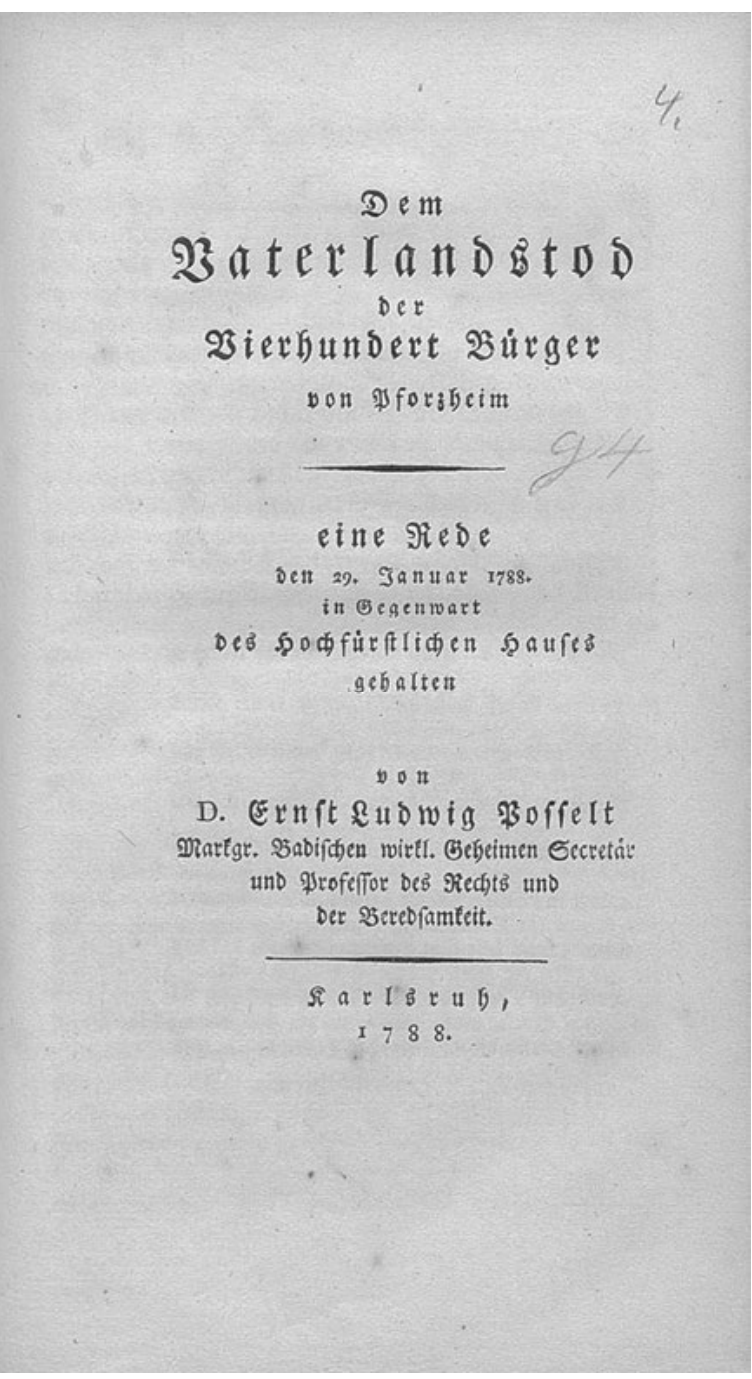

Abb. 2: Ernst Ludwig Posselt: Dem Vaterlandstod der Vierhundert Bürger von Pforzheim, Karlsruhe 1788, Titelblatt.

Posselt verdankt Büchner etwa die comparatio mit den großen antiken Vorbildern: So vergleicht Posselt die Pforzheimer Bürger mit zwei legendären heroischen Selbstaufopferungen der Antike: der dreihundert Spartaner unter Leonidas bei

rung 3, Heft 5, Leipzig 1788, S. 462-482; Ernst Ludwig Posselt: D. Ernst Ludwig Posselt's kleine Schriften, Nürnberg 1795, S. 183-214; Ernst Ludwig Posselt: Erinnerungen an die Schlacht bei Wimpfen und den Tod der vierhundert Pforzheimer. Enthaltend die Geschichte der Schlacht von Ernst Münch und die Gedächtnißrede auf die Gefallenen, hrsg. von C. V. Sommerlatt, Freiburg 1824, S. 17-36; Gemälde der Wirklichkeit alter und neuer Zeiten, in interessanten Begebenheiten aus der Geschichte gesammelt, zur angenehmen und nützlichen Lectüre für Jedermann, Freiburg 1830, S. 124-146. Zu Posselts großer Bedeutung für die Spätaufklärung in Südbaden vgl. Wilhelm Kühlmann: Facetten der Aufklärung in Baden. Johann Peter Hebel und die Karlsruher ,Lateinische Gesellschaft', Freiburg [u.a.] 2009. 
den Thermopylen 480 v. Chr. sowie der vierhundert Römer bei Kamarina auf Sizilien im Ersten Punischen Krieg 258 v. Chr. Mit diesen Vergleichen begründet Posselt zugleich eine translatio heroica, den Transfer eines heroischen Nationalcharakters von Griechenland über Rom nach Deutschland. ${ }^{25}$ In Posselts Rede figurieren die Pforzheimer als Muster aufgeklärter Bürger in einer konstitutionellen Monarchie, dementsprechend wird auch der Fürst in die Pflicht genommen, vom Tyrann unterschieden und aufs Gesetz verpflichtet. Mehr noch: Die vierhundert Pforzheimer Bürger verkörpern in ihrer Fürsten- und Verfassungstreue das identitätsstiftende Muster einer Nationaltugend, das Posselt wie einen Gründungsmythos für einen deutschen Staatenbund oder Nationalstaat propagiert.

Posselt seinerseits verdankt den Stoff seiner Rede dem Drama eines Pforzheimer Handelsmanns und Dichterdilettanten, Ernst Ludwig Deimling: „Die Vierhundert Pforzheimer Bürger oder die Schlacht bey Wimpfen“ (1788, Abb. 3). Deimling hatte sich, angeregt durch de Belloys Trauerspiel „Le siège de Calais“, vorgenommen, „dem französischen Drama: die Belagerung von Calais ein teutsches entgegen zu sezen $[\ldots]^{\text {“. }: 26}$

,ich [Deimling] [...] wünschte, ein Gegenstück aus der teutschen Geschichte aufzufinden. Da fiel mir die von mir selbst fast vergeßene Grosthat der 400 Pforzheimer Bürger bei, und ich nahm sogleich vor, einen Versuch zu wagen, diesen Heroismus zu dramatisiren. ${ }^{\text {"27 }}$

Die kulturpatriotische Absicht, ein deutsches Pendant zu dem auch in Deutschland populären französischen Nationaldrama zu schaffen, zeigt sich in dem Untertitel: „Vaterländisches Trauerspiel in fünf Aufzügen“. Deimlings Drama erschien zwar erst nach Posselts Rede im gleichen Jahre 1788, lag aber als Manuskript spätestens 1784 vor, wie neben der Widmung an den regierenden badischen Markgrafen Carl Friedrich die ausführliche Geschichte der Entstehung in einer Nachschrift bezeugt. ${ }^{28}$

25 Posselt: Dem Vaterlandstod der Vierhundert Bürger von Pforzheim (Anm. 25), S. 232.

26 Ernst Ludwig Deimling: Die Vierhundert Pforzheimer Bürger, oder Die Schlacht bey Wimpfen. Ein vaterländisches Trauerspiel in fünf Aufzügen nebst Vorbericht, eine kurze Geschichte von Pforzheim und die Veranlassung zu diesem Unternehmen enthaltend, Karlsruhe 1788. Ich zitiere nach der zweiten, titel- und textidentischen Ausgabe (Druck: Pforzheim und Augsburg: E. Kletts Wittwe und Franck 1788, S. I), die vielleicht eine überregionale Verbreitung des patriotischen Trauerspiels fördern sollte.

27 Deimling: Die Vierhundert Pforzheimer Bürger (Anm. 27), S. 176.

28 Vgl. Deimling: Die Vierhundert Pforzheimer Bürger (Anm. 27), Widmung und Nachschrift, S. 171-185. Die Widmung, die vierzehn ungezählte Seiten umfasst, datiert vom „15. Feb[ruar] 1785“, die „Nach-Schrift“ vom „5. Aprill 1788“. Der Nachschrift voraus geht eine Deimling gewidmete elegische Dichtung auf den Heldentod der Pforzheimer in lateinischer Sprache von „M. C. P. F. K.“ (S. 161-170). In der Nachschrift erläutert Deimling, dass die Schrift durch Vermittlung der Hofräte Wieland, Ring und Molter dem Fürsten zur Kenntnis gebracht wurden. Im Dezember 1787 habe ihm dann Ernst Ludwig Posselt mitgeteilt, dass er im Auftrage des Fürsten im Januar 1788 das historische Ereignis in einer Gedenkrede würdigen würde, um „diesen bis in Tod getreuen Männern ein verdientes Denkmal zu errichten“, ebd., S. 178-180. Im Pränumerantenverzeichnis, das dem Drama beige- 


\section{Die Biergunder \\ \$forzheimer Şürger, \\ D D E}

Die Schladit bey æimpfen

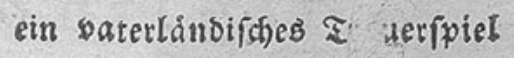

it

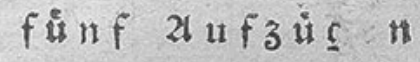

$$
\begin{aligned}
& \text { nebit } \\
& \text { Dorbertdis }
\end{aligned}
$$

eine futge Befchichte von Sforjgcim uns bie

Beranlafung zu diefen unternegmen entgaitend.

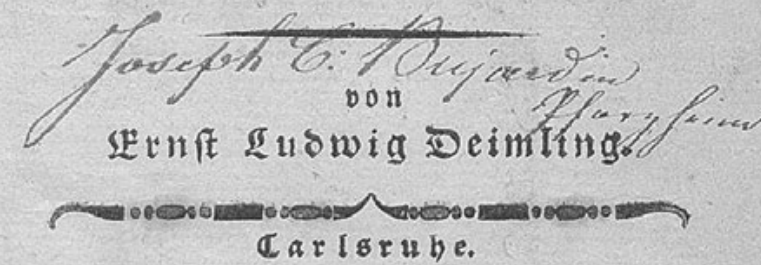

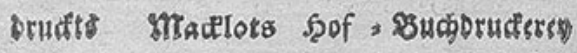

\& $78 \&$
Abb. 3: Ernst Ludwig Deimling: Die vierhundert Pforzheimer Bürger, oder Die Schlacht bey Wimpfen [:] ein vaterländisches Trauerspiel in fünf Aufzügen, Karlsruhe 1788, Titelblatt.

Deimlings Prosadrama, von der überregionalen Kritik als „seichte[s] Product[]“ abgetan, genügt immerhin den aristotelischen Einheiten: Dargestellt ist die Schlacht bei Wimpfen, freilich ausschließlich durch Botenbericht vermittelt, Handlungszeit ist der 6. Mai 1622, Schauplatz das Hauptquartier des markgräflichen Heeres. Neben den abgehenden und zurückkehrenden Heerführern dominieren die Szene aber zwei Frauen, die sich in dem Lager aufhalten: die Frau des Bürgermeisters und die Frau eines Pforzheimer Hauptmanns. Sie erläutern langatmig die Vorgeschichte und bereiten sich innerlich auf den Tod ihrer Mitbürger vor, der sich sukzessiv ereignet. Nachdem die ersten „30 Mann von denen Pforzheimern“ gefallen sind,

bunden ist, figuriert Ernst Ludwig Posselt neben Wilhelm Ludwig von Baden-Durlach, dem Bruder des Regierenden Markgrafen. 
entkräftet Sophie, die Frau des Bürgermeisters, Margrets Kummer über die „armen Witwen und Waisen“, indem sie den Tod „für Fürsten und Vaterland und Gewissens-Freiheit" verklärt:

MARGRET.

Ach, die armen Witwen und Waisen!

SOPHIE.

Für diese wird GOtt sorgen! die Männer starben als Helden auf dem Bette der Ehren. Was kann man sich schöners denken, als der allgemeinen Sage, seinem Fürsten und Vaterland und Gewissens-Freiheit sein Leben aufgeopfert, und so viel zum Sieg beigetragen zu haben?29

Überdies rückt auch schon Deimling den Heldentod der Pforzheimer in eine typologische Tradition: So wird der Römer gedacht, die sich der karthagischen Armee opferten, und so alludieren die ziemlich langen letzten Worte des Bürgermeisters Deimling, der auf den Tod verwundet ins Hauptquartier gebracht wird und im Beisein des gerührten Tilly stirbt, im charakteristischen „Sage“ das heroische Spartiaten-Epigramm: ${ }^{30}$

BURGERMEISTER.

Grüße und küsse - meine Kinder! - Segne sie in meinem Namen, auf alle - ihre Nachkommen! - sage ihnen: - daß sie, wie ich - ihrem Fürsten - getreu - bis in Tod dienen [...] - Sage dem Marggraven! - daß ich - ihm getreu - und für ihn - willig - und freudig gestorben sey. ${ }^{31}$

Das historische Ereignis, das Georg Büchners Schulrede in der Nachfolge von Posselts Rede, die ihrerseits auf Deimlings Drama fußt, behandelt, ist die Schlacht bei Wimpfen am 6. Mai 1622. Das protestantische Heer unter Führung des lutherischen Markgrafen Georg Friedrich von Baden-Durlach erlitt hier eine vernichtende Niederlage gegen die bayerisch-ligistische Armee unter General Tilly und dem spanischen Feldherrn Gonzalo Fernández de Córdoba. Volltreffer in zwei Munitionswagen hatten das ohnehin zahlenmäßig unterlegene markgrälliche Heer, das in den Neckarauen vor Wimpfen eine Wagenburg errichtet hatte, entscheidend geschwächt. Die enorme Pulverexplosion, die auch die zeitgenössischen Schlachtabbildungen wiedergeben - wie Merians Karte vom Schlachtfeld (Abb. 4) -, nötigte den Markgrafen zur Flucht. Die späteren Historiographen haben neben Pforzheimer Archivalien ausschließlich auf den Bericht im „Theatrum Europaeum“ als Quelle für den angeblichen „Helden-Tod der vierhundert Pforzheimer“ hingewiesen. Doch die Schlacht von Wimpfen im Mai 1622 war ein großes Medienereignis, das in zahlreichen zeitnahen Flugschriften und Einblattdrucken überliefert ist. Darin ist zwar von heroischem Widerstand und „HeldenMuth“, aber von keinen

29 Deimling: Die Vierhundert Pforzheimer Bürger (Anm. 27), III, 2, S. 85.

30 Vgl. zur regen Rezeption des Spartiaten-Epigramms im 18. Jahrhundert in Deutschland die instruktive Studie von Albertz: Exemplarisches Heldentum (Anm. 12), besonders S. 174-175.

31 Deimling: Die Vierhundert Pforzheimer Bürger (Anm. 27), V, 5, S. 153. 


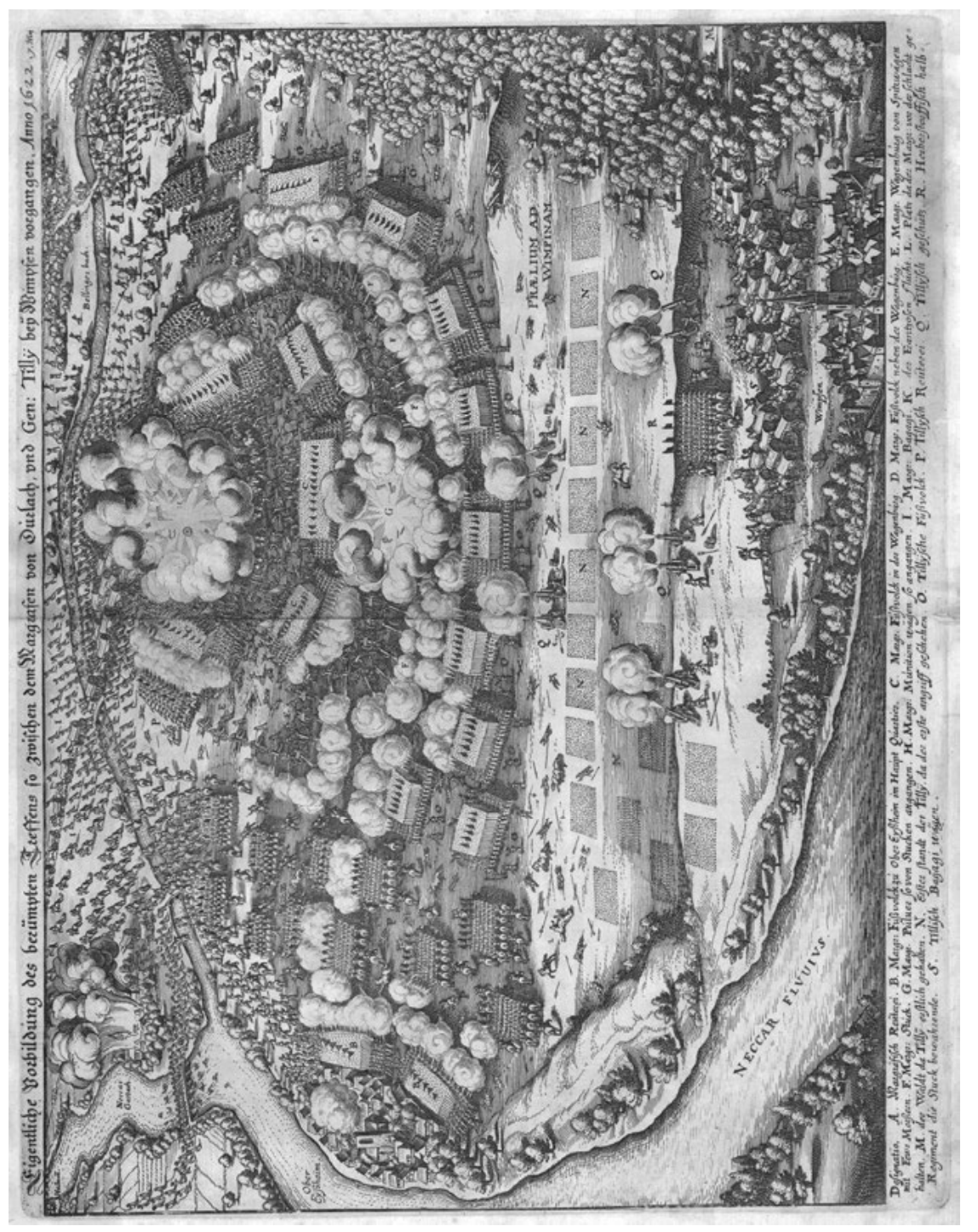

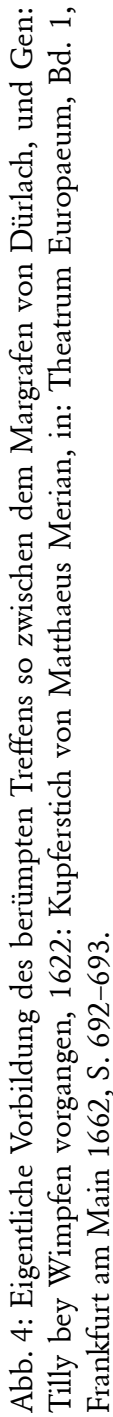


Pforzheimern die Rede. ${ }^{32}$ Es wird lediglich erwähnt, dass sich „der Obriste Heldmstädter mit dem weissen Regiment bis auf den letzten Mann [...] gewehrt" ${ }^{\times 33}$ habe. Diese Wendung findet sich auch in weiteren Beschreibungen der Schlacht.

Wann und durch wen das ,weisse[] Regiment“, die vier heldenhaften Fähnlein, etwa vierhundert Soldaten, die den Rückzug des Markgrafen gedeckt haben, mit den vierhundert Pforzheimer Bürgern identifiziert wurden, lässt sich nicht zweifelsfrei klären. Im Jahre 1770 erwähnt Johann Christian Sachs (1720-1789), Rektor des Karlsruher Gymnasium illustre, in seiner mehrbändigen „Geschichte der Marggravschaft [...] Baden“, dieses Faktum als mündliche Überlieferung:

„Man meldet, daß bey 400. Mann von der Burgerschaft zu Pforzheim, welche dem Marggraven zu einer Leibgarde gedient hätten, fast bis auf einen Mann sich haben niederhauen lassen." ${ }^{\text {"34 }}$

Doch erst Deimlings Drama und Posselts mustergültige Rede aus dem Jahre 1788 machten die historisch fragwürdige Heldentat der vierhundert Pforzheimer Bürger mit einem Schlag deutschlandweit bekannt. Das badische Herrscherhaus vereinnahmte den Kult für sich, indem es im Jahre 1822 eine „Feyer der vor 200 Jahren bey Wimpfen für Fürst und Vaterland gebliebenen 400 Pforzheimer" förderte, in deren Rahmen Deimlings Drama zum ersten Mal aufgeführt wurde. ${ }^{35}$ Das „Programm zur Feyer" sakralisiert in spezifischer Weise das Heldengedenken, indem es das Totengedächtnis mit einem Trauergottesdienst verbindet.

Mit der Zweihundertjahrfeier der Schlacht bei Wimpfen setzte ein regelrechter Kult ein, in dem der Opfertod des bürgerlichen Heldenkollektivs rednerisch, poetisch und bildkünstlerisch verklärt wurde. ${ }^{36}$ Doch bereits Mitte des 19. Jahrhun-

32 Vgl. Wilhelm von Sitzingen [Pseudonym]: HeldenMuth / Der thewren hochwerthen Ritter / Cavallieren und Soldaten Gegenwehr / ernstlicher Schlacht / oder Treffens vor Wümpffen, o. O. 1622. Vgl. Moritz Gmelin: Beiträge zur Geschichte der Schlacht bei Wimpfen. (6. Mai 1622), in: Zeitschrift für die Geschichte des Oberrheins 31, 1879, S. 332-448; 32, 1880, S. 1-56, hier S. 15-16, Nr. 9. Gmelin gibt den immer noch besten, wenn auch nicht vollständigen Überblick über die zeitgenössische Rezeption der Schlacht bei Wimpfen.

33 Matthaeus Merian: Theatrum Europaeum oder Ausführliche und Wahrhaftige Beschreibung aller und jeder denckwürderiger Geschichten, Frankfurt am Main 1662, S. 627.

34 Johann Christian Sachs: Einleitung in die Geschichte der Marggravschaft und des marggrävlichen altfürstlichen Hauses Baden. Vierter Theil, Karlsruhe 1770, S. 433, Anm. y. Die Wendung „bis auf den letzten Mann“ meint „Alle“; vgl. Karl Hofmann: Aus badischen Landen. Beiträge zur Heimatsgeschichte, Weinheim/Leipzig 1917, S. 27.

35 Vgl. Editionsbericht der MA, Bd. 1, 2, S. 46.

36 Wenigstens erwähnt sei das Ehrendenkmal in der Pforzheimer Schloss- und Stiftskirche St. Michael von 1834 sowie die poetischen Bearbeitungen der Sage von Anton Dietrich, Karl Fernand, Eduard Brauer und Adolf Bube. Vgl. Anton Dietrich: Heldentod der vierhundert Bürger von Pforzheim in der Schlacht bei Wimpen am 6. Mai 1622, in: Stuttgarter Morgenblatt 1822, Nr. 123; Karl Fernand: Die Schlacht bei Wimpfen. Ein vaterländisches Heldenlied, Karlsruhe 1838; Eduard Brauer: Die vierhundert Pforzheimer, in: Eduard Brauer (Hrsg.).: Sagen und Geschichten der Stadt Baden im Grossherzogthum und ihrer näheren und entfernteren Umgebungen in poetischem Gewande, Karlsruhe [1845], S. 144-147; Adolf Bube: Die Pforzheimer Bürger, in: August Schnelzer (Hrsg.): Badisches Sagen-Buch. Eine Sammlung der schönsten Sagen, Geschichten, Märchen und Legenden 
derts wurde der Wahrheitsgehalt der Episode bezweifelt, und spätestens seit den Studien von David Coste (1874) und Karl von Reitzenstein (1891) kann sie nicht mehr als historisches Faktum gelten. ${ }^{37}$ Seitdem das vermeintliche Selbstopfer der vierhundert Pforzheimer Bürger ins Reich der Legende verwiesen worden ist, hat die historische Forschung das Thema ad acta gelegt. Doch für die Frage nach Krisen und Konjunkturen des Heroischen ist nicht entscheidend, ob eine Heldentat wahr oder erfunden ist, sondern warum sie zu bestimmten Zeiten aufkam und als glaubwürdig verbreitet wurde.

So ist es sicher kein Zufall, dass Deimlings ,vaterländisches Trauerspiel“ und Posselts Gedenkrede den Opfertod des bürgerlichen Heldenkollektivs am Vorabend der Französischen Revolution zum wehrhaften Muster eines Verfassungspatriotismus in einer konstitutionellen Monarchie verklärt haben. Und was bedeutet der Entstehungskontext für Büchners Rede im Jahre 1829, am Vorabend der Julirevolution? Wie viele Mitschüler und Lehrer am Darmstädter Pädagog sympathisierte Büchner mit den Ideen der Französischen Revolution und der Abschaffung der Bourbonenherrschaft im Juli 1830. Diese revolutionäre Tendenz ist mindestens latent in der Wahl und Durchführung des Themas zu erkennen: ein bürgerliches Heldenkollektiv, das den „Welterlöser-Tod“ für das Vaterland wählt. Und es ist auch kein Zufall, dass Büchner den „Helden-Tod“ der Pforzheimer Vierhundert - dieser Vergleich ist ganz ungewöhnlich - mit einem heroischen Selbstopfer der Französischen Revolutionsarmee vergleicht. Diese Gleichsetzung passt zu dem jakobinischen Motto Bürgers, liegt allerdings sowohl mit der nationalen Sinngebung als auch mit dem Motiv der Fürstentreue im Widerstreit - beides Anleihen bei Posselt. Die imaginäre Rede der Gefallenen an die Zeitgenossen gestaltet Büchner aber deutlich parteiischer als Posselt, und der politische Schluss mit dem Imperativ „Wählet“ aktualisiert die Episode stärker und fordert das Publikum zur Entscheidung und zum Handeln auf.

Nur wenige Jahre später wich freilich Büchners Begeisterung für das Heroische einer ebenso gründlichen Ernüchterung. Vor dem Hintergrund des im Brief vom 10. März 1838 an seine Braut evozierten grässlichen „Fatalismus der Geschichte ${ }^{\text {38 }}$ verabschiedet er sich von der Vorstellung des Helden als eines Agens und Lenkers der Geschichte. „Dantons Tod“ (1835) inszeniert vielmehr auf eindrucksvolle Weise gerade die Ohnmacht des Einzelnen, der dem Geschichtslauf hilflos

des Badischen Landes aus Schrifturkunden, dem Munde des Volkes und der Dichter, Bd. 2, Karlsruhe 1846, S. 391-395.

37 Zwar gab es noch Ende des 19. Jahrhunderts Versuche, wie etwa durch den Stadtpfarrer Brombacher, die Kriegsepisode historisch zu beglaubigen. Friedrich Brombacher: Der Tod der 400 Pforzheimer bei Wimpfen nicht eine Sage, sondern eine Thatsache: genaue Untersuchung der Streitfrage auf Grund des ältesten hiesigen Taufbuches mit Benützung der ältesten geschichtlichen Quellen, Pforzheim, Selbstverl. des Verf., 1886.

38 Georg Büchner: Werke und Briefe. Nach der historisch-kritischen Ausgabe von Werner R. Lehmann, kommentiert von Karl Pörnbacher, Gerhard Schaub, Hans-Joachim Simm und Edda Ziegler, München/Wien 1980, S. 256, Nr. 18. 
ausgeliefert ist. Sogar der passive Heroismus der stoischen Tradition wird abgelehnt. Die im Brief ebenfalls evozierte „entsetzliche Gleichheit“ „in der Menschennatur" 39 lässt nämlich selbst die heroische Haltung der apatheia als eine theatralische Pose erscheinen, welche das kreatürliche Moment des Leids negiert. ${ }^{40}$

\section{Abbildungsnachreeise}

Abb. 1: Klassik Stiftung Weimar.

Abb. 2: Digitale Sammlung der Staatsbibliothek zu Berlin, http://digital. staatsbibliothek-berlin.de/werkansicht/?PPN=PPN717592774, 5. Oktober 2015.

Abb. 3: Digitale Sammlung der Staatsbibliothek zu Berlin, http://digital. staatsbibliothek-berlin.de/werkansicht/?PPN=PPN750626348\&LOGID =LOG_0006, 5. Oktober 2015.

Abb. 4: Digitale Sammlungen der Universität Augsburg.

39 Ebd.

40 „CAMILLE. [...] schreit nur und greint, wie es euch ankommt! Schneidet nur keine so tugendhafte und so witzige und so heroische und so geniale Grimassen, wir kennen uns ja einander, spart euch die Mühe! / HÉRAULT. Ja, Camille, wir wollen uns beieinandersetzen und schreien; nichts dummer, als die Lippen zusammenzupressen, wenn einem was weh tut. Griechen und Götter schrien, Römer und Stoiker machten die heroische Fratze. / DANTON. Die Einen waren so gut Epikureer wie die andern. Sie machten sich ein ganz behagliches Selbstgefühl zurecht. Es ist nicht so übel, seine Toga zu drapieren und sich umzusehen, ob man einen langen Schatten wirft. Was sollen wir uns zerren? Ob wir uns nun Lorbeerblätter, Rosenkränze oder Weinlaub vor die Scham binden oder das häßliche Ding offen tragen und es uns von den Hunden lecken lassen?“, ebd., S. 64. 
